\title{
Crystal Orientation Mapping via STEM/NBD: Improved Quality with Precession Electron Diffraction and Energy Filtering
}

\author{
G. Pavia, * G. Benner, * H. Niebel, * E.F. Rauch** and M. Véron** \\ * Carl Zeiss NTS, Carl Zeiss Strasse 56, 73447 Oberkochen, Germany \\ ** SIMAP laboratory, 101 rue de la Physique, 38402 Saint Martin d'Hères Cedex, France
}

The research on materials, both organic and inorganic, is in a phase of transition from microscale to nanoscale. The investigation techniques developed for investigating materials at the microscale appear very often not suitable anymore for the investigation at the smaller scale. This holds particularly in the field of crystals, where techniques like X-Ray Diffraction (XRD) for structure solving and Electron Backscatter Diffraction (EBSD) for crystal orientation mapping are limited by their intrinsic spatial resolution. Extending these techniques to Transmission Electron Microscopy (TEM) is an answer to the demand for improved spatial resolution. The present work will focus on crystal orientation mapping.

This kind of mapping requires a fast acquisition technique and a reliable analysis method. Both the acquisition and the post-processing are necessary to enable the automation of the mapping. Since some years such kind of packages are available. In particular the so-called Automatic Crystal Orientation Mapping (ACOM) and Automatic Crystal Orientation and Phase Mapping (ASTAR) tools experience currently a fast dissemination worldwide. These tools are set up to scan a focussed beam in TEM/NBD (Nano Beam Diffraction) mode and consequently work with TEMs not equipped with the STEM option.

Instead, the following results are acquired using a Zeiss Libra $200 \mathrm{FE}$ operated in STEM/NBD mode, which allows both the diffraction patterns (DP) and large area (STEM) images to be acquired under the same microscope configuration. (Fig. 1)

The potentiality of the present operating mode is evaluated by considering the misindexing problem related to the famous $180^{\circ}$ orientation ambiguity that frequently occurs for zone axis DPs. It is confirmed that the combination of orientation mapping with Precession Electron Diffraction (PED) allows the extent of this ambiguity problem to be dramatically reduced (Fig. 2.a and b). This work also demonstrates for the first time that Zero Loss Filtering (ZLF) will have a similar beneficial effect on the indexing quality (Fig. 2.c). Of interest is the fact that using in column energy filtering is straightforward as it only requires the energy window (slit) to be inserted.

The beneficial effect of PED is known to be related to the higher number of reflection excited when the beam is precessed [1] (compare Fig. 3.a and b). By contrast, ZLF improves the indexing for a different physical reason: a close examination of the indexing procedure shows that it is the significant increase of the signal over background noise ratio in the DP that helps the diffracting spots to be unambiguous recognized.(Fig. 3.c)

It is worth emphasizing that, thanks to the large angular acceptance of the corrected in-column Omega filter, PED and ZLF can be used either independently or in a combined mode.

[1] E.F. Rauch et al., Special Issue on "Precession Electron Crystallography" of Zeits. Krist. $225,(2010)$ issue $2-3$ 

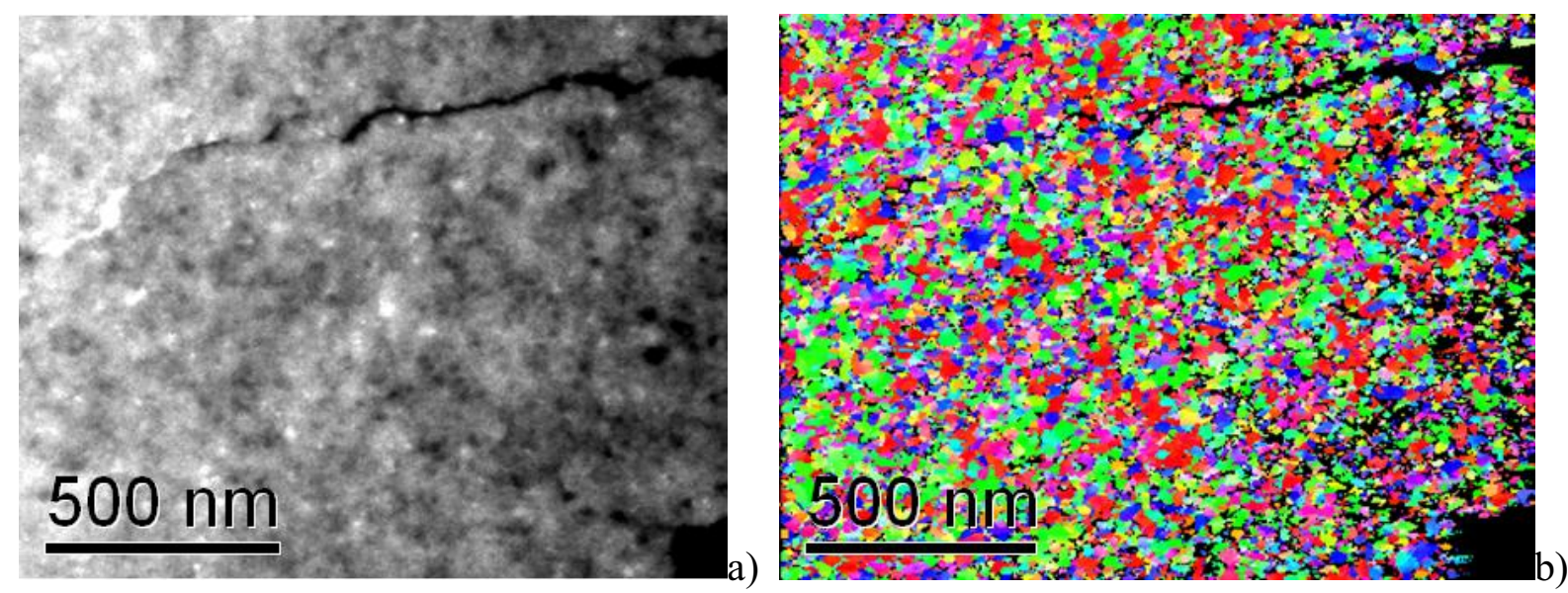

Fig. 1: a) STEM/NBD image from a Ni-Fe sample; b) orientation mapping from the same region
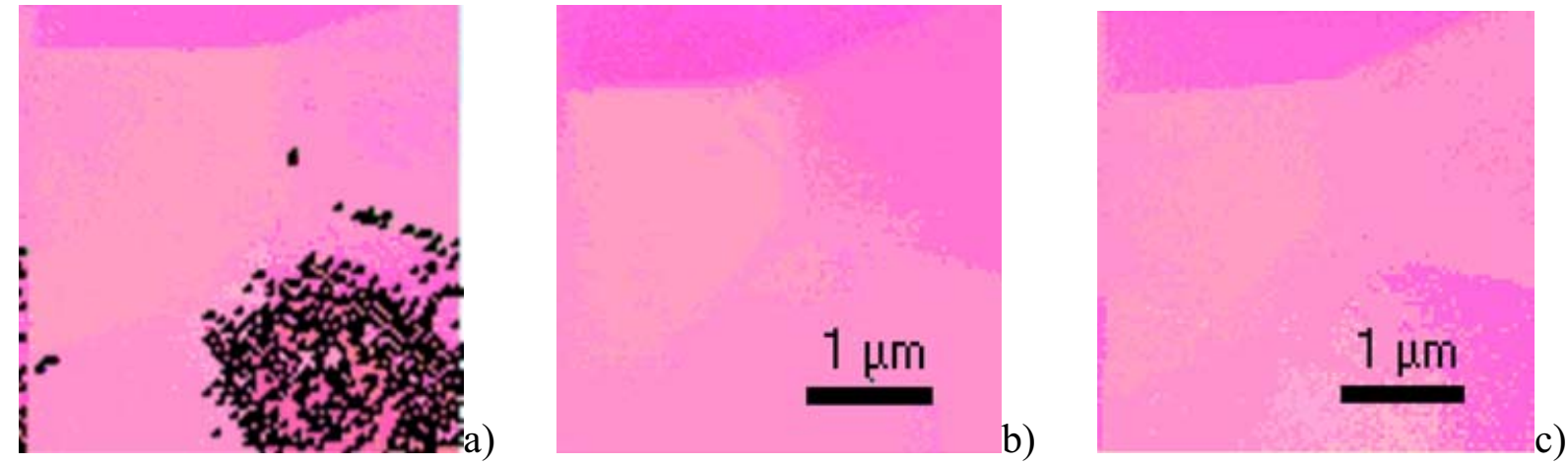

Fig. 2: Virtual bright field: a) without precession or energy filtering; b) with $0.75^{\circ} \mathrm{PED}$; c) with Zero Loss Filtering (40 eV slit). The black dots in Fig. 1.a) indicates were the $180^{\circ}$ ambiguity is present. In Fig 1.b) and 1.c) the ambiguity is resolved.
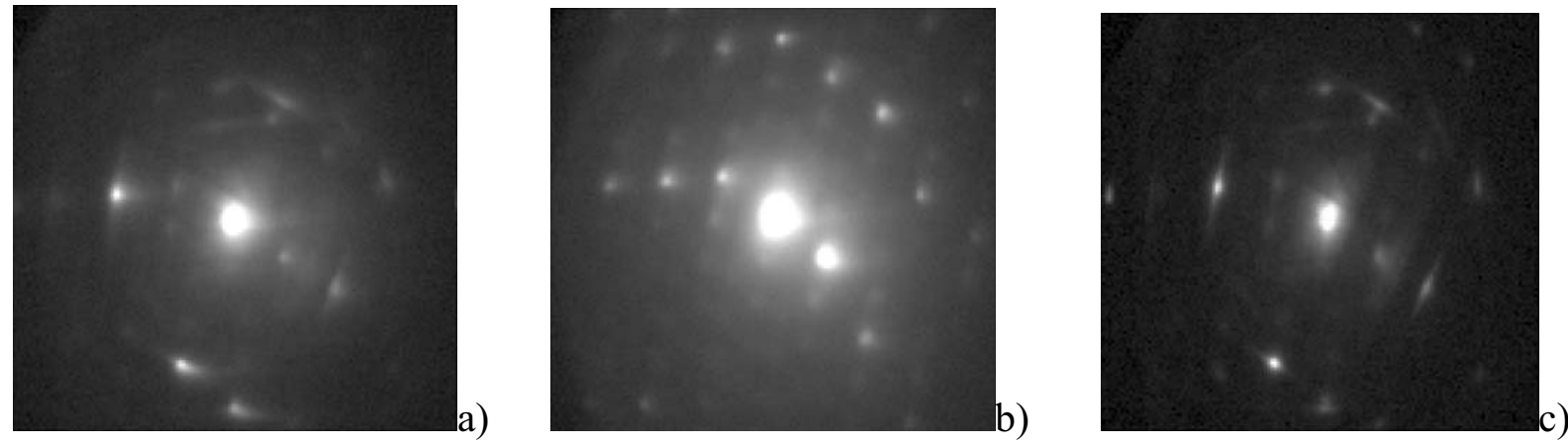

Fig. 3: NBD patterns from ASTAR acquisitions: a) conventional; b) with $0.75^{\circ}$ precession; c) energy filtered with $40 \mathrm{eV}$ slit width. Spots from the first order Laue zone are visible only in 2.b) and 2.c) 